Geograficando, vol. 13, n. ${ }^{\circ}$ 2, e027, diciembre 2017. ISSN 2346-898X

Universidad Nacional de La Plata.

Facultad de Humanidades y Ciencias de la Educación.

Departamento de Geografía

\title{
La evolución del mapa social del Gran San Miguel de Tucumán. Un estudio del periodo 1991 a 2010 a través del análisis factorial
}

\author{
Evolution of the social map of the Greater San Miguel de Tucuman \\ area. A research of 1991/2010 PERIOD through factorial analysis
}

\section{Ana Ester Batista Zamora* Juan José Natera Rivas**}

\author{
* Departamento de Geografía. Facultad de Filosofía y Letras. Universidad de Málaga, España \\ **Departamento de Geografía. Facultad de Filosofía y Letras. Universidad de Málaga, España | \\ **jjnatera@uma.es
}

\section{PALABRAS CLAVE}

Ecología factorial

Crecimiento urbano

Fragmentación urbana

\section{KEYWORDS}

Factorial ecology

Urban growth

Urban fragmentation

\section{RESUMEN}

El aglomerado del Gran San Miguel de Tucumán, capital de la Región Noroeste de la República Argentina, ha experimentado un notable aumento de población desde 1991. Dicha población dista de ser homogénea en lo relativo a sus niveles de instrucción, estructura por edad o características de sus viviendas, entre otras, al tiempo que el aumento del parque habitacional ha tenido lugar entre dos extremos: urbanizaciones cerradas y barrios de habitat popular, en muchas ocasiones con problemas dominiales. En este contexto, y aplicando análisis factoriales de componentes principales a 13 variables censales, correspondientes a los Censos de población de 1991, 2001 y 2010, mostramos cómo las causas subyacentes a la distribución espacial de la población se han mantenido muy estables durante el periodo analizado, al tiempo que su distribución espacial refleja un proceso de progresiva polarización y problemas habitacionales no resueltos. 


\section{Introducción}

El aglomerado del Gran San Miguel de Tucumán ha experimentado un importante aumento de su población en los últimos 20 años, pasando de los 622.324 censados en 1991 a los 794.327 en 2010, lo que le sitúa como el más importante de la Región Noroeste de la República Argentina, tanto por población como por volumen de actividades económicas y centralidad (el aglomerado es uno de los cinco nodos nacionales identificados en el Atlas ID -Ministerio, 2016-). Su población dista de ser homogénea: presenta marcadas diferencias en lo relativo a los niveles de bienestar (atendiendo al Índice de Privación Material de los Hogares, a comienzos de siglo coexistía un 50,2\% de hogares sin privación con un 19,2\% de hogares con privación convergente), activos de instrucción (en 2010 el 56\% de la población que ya no asiste a establecimientos educativos tiene como máximo nivel de instrucción la secundaria incompleta, frente al 14\% que cuenta con estudios superiores), o características básicas de sus viviendas (el 13\% de la población ese mismo año no contaba con retrete con descarga de agua), entre otras, habiéndose registrado también dinámicas diferentes en los procesos de aparición de tipos de hábitat contrapuestos (villas miseria y barrios cerrados, en los extremos de las características habitacionales -Malizia \& Boldrini, 2012-).

Estas diferencias tienen una vertiente espacial: la población tiende a estar diferenciada en el tejido urbano según determinadas variables referidas no sólo a ella misma, sino también a los hogares que conforman y a las viviendas en las que habitan. A modo de ejemplo, si representamos la información censal a nivel de radio censal, puede observarse cómo los hogares sin retrete se localizan en lugares diferentes con respecto a aquellos residentes en departamentos, o que la población mayor de 64 años se encuentra de forma mayoritaria en el centro de la ciudad, mientras que la población infantil lo hace en la periferia.

Este tipo de hechos no sólo está presente en el aglomerado tucumano, sino que es extrapolable a la práctica totalidad de ciudades por encima de un cierto volumen de población. Es más, el estudio de la diferenciación socioespacial de la población en la ciudad latinoamericana es un tema de investigación abordado en numerosas ocasiones, y que ha generado un apreciable número de modelos. En una primera fase construidos en su mayoría a partir de información cualitativa, los encuadrables en la "escuela" norteamericana (Griffin \& Ford, 1980; Howell, 1989) y en la “escuela” alemana (Bähr \& Mertins, 1982; Borsdorf, 2003; Janoschka, 2002; Mertins, 1995), son tal vez los más conocidos; y, gracias al progresivo acceso a información estadística espacialmente muy desagregada, han ido apareciendo incursiones en el tema que, de una u otra forma, han tratado de obtener áreas de la ciudad diferenciadas a partir de variables cuantitativas referidas a la población, los hogares y las viviendas (al respecto, cfr. especialmente a Buzai, 2014).

Este último conjunto de investigaciones se centra en dilucidar qué elementos subyacen en la distribución espacial de la realidad visible aprehendida a través de la información censal, en la línea que ya iniciaran Shevky y Bell en los años cincuenta del siglo pasado, continuando a través de la ecología factorial (Timms, 1976), y que ha conocido cierto florecimiento en la realidad argentina en los últimos años. Nuestro objetivo está en relación con esta última corriente, y consiste en responder a las siguientes preguntas: ¿cuáles son las dimensiones principales que subyacen a la diferenciación socioespacial de la población censada en los Censos de 1991, 2001 y 2010 en el Gran San Miguel de Tucumán? Y, una vez identificadas, ¿̇se han mantenido estables en los veinte años que separan las tres operaciones censales? 
La pertinencia de estas cuestiones se pone de manifiesto al atender, aunque sea de manera muy sucinta, a las características básicas del proceso de expansión reciente del aglomerado. Tal y como se indica en GEO (2016), su crecimiento experimentó un punto de inflexión en la década del sesenta del siglo pasado; hasta fines de los cincuenta el crecimiento el tejido urbano mantenía cierto orden, en el contexto del mantenimiento del trazado en damero fundacional, pero desde entonces el crecimiento, tanto en lo vertical como en lo horizontal, rompe esa lógica. Entre los sesenta y los ochenta el aglomerado crece a través de proyectos de vivienda de iniciativa estatal, insertos, contiguos, o incluso desprendidos del tejido urbano preexistente. Paralelamente, se registra un aumento de asentamientos irregulares, al margen de cualquier control o planificación. Esta situación fue modelizada por Mertins (1995), quien en su modelo del Gran San Miguel de Tucumán identificaba las áreas central y occidental como compuestas por viviendas de clases media alta y alta, y el resto del aglomerado donde las viviendas eran de clase media y baja.

A partir de la década de los noventa el Estado se retira como productor de vivienda, y las desigualdades se incrementan: a una urbanización localizada en áreas con escasa aptitud para ello, no siempre legal, y protagonizada por los sectores menos pudientes, se contrapone otra protagonizada por el espectro contrario. Esta toma la forma de conjuntos autocontenidos, localizados en el municipio de Yerba Buena (que aparece, como los principales hitos mencionados en el texto, en la figura 1). Y en cuanto a la obra pública llevada a cabo en el aglomerado en el último decenio, tendió a profundizar la fragmentación socioespacial ya existente, puesto que la elección de las grandes extensiones de terreno para levantar la vivienda de iniciativa estatal no tendió a la equidistribución de grupos sociales para tratar de contrarrestar fenómenos sociales desfavorables (Gómez, Cuozzo y Peralta, 2015).

Figura 1. Gran San Miguel de Tucumán (2010). Localización de los principales hitos citados en el texto.

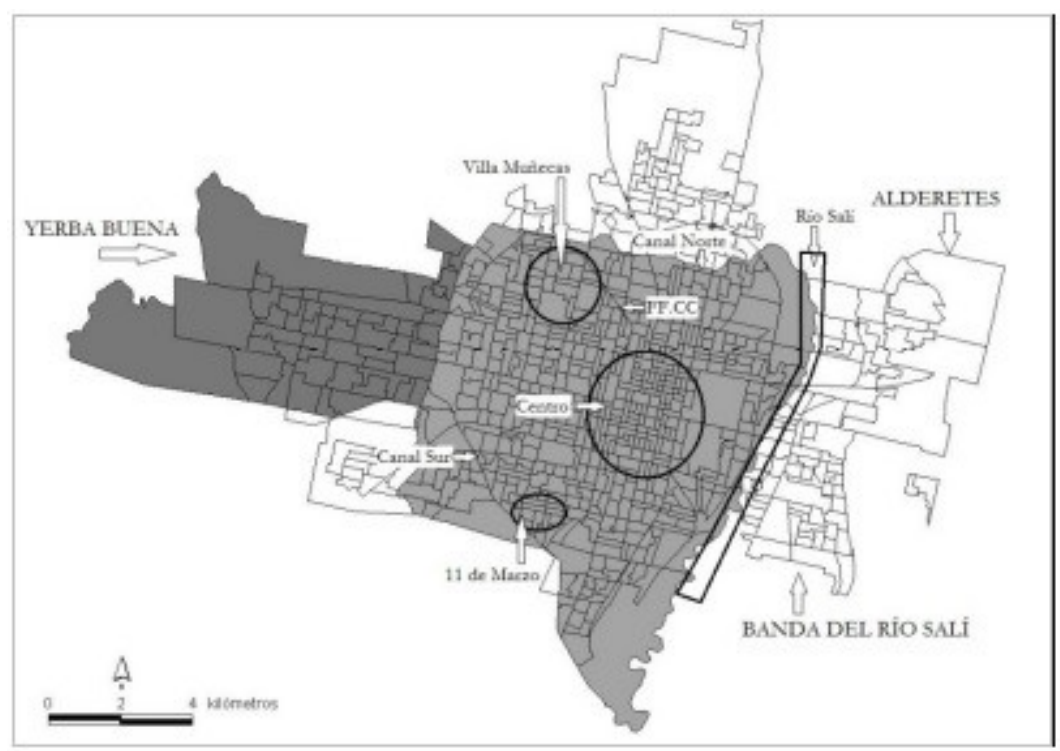

Elaboración propia.

El proceso de expansión del Gran San Miguel en nuestro periodo de estudio ha estado, por tanto, caracterizado por una notoria desigualdad en la dotación infraestructural de los diferentes barrios, 
críticamente reducida en la práctica en la totalidad de la periferia del aglomerado, siendo el más vulnerable el sector sur. Esta situación se combina con otra de irregularidad dominial, realidad especialmente grave en las áreas colindantes con los Canales Sur y Norte, las vías del ferrocarril y el sector oriental de San Miguel, que ha llegado a ser definido como un sector "que no existe", por sus gravísimas condiciones de abandono y marginalidad (Salas, 2016).

Esta realidad contrasta no sólo con la del centro, el área mejor dotada infraestructuralmente y corazón funcional del Gran San Miguel, sino con el municipio de Yerba Buena, en el extremo oriental del aglomerado. Originalmente era una zona de viviendas secundarias de la clase alta tucumana que, atraída por las comparativamente buenas condiciones ambientales, desde los setenta ha venido conociendo un progresivo proceso de consolidación, en el que la presencia de barrios marginales y actuaciones públicas de vivienda para clases bajas han sido testimoniales. Un proceso de consolidación y crecimiento en el que los grandes protagonistas han sido, desde la década del 2000, las urbanizaciones cerradas. Son reflejo del crecimiento formal del espacio construido, (Geo, 2006) en el que predominan los intereses sectoriales sobre el interés común, y la iniciativa privada a través de la oferta de "verde, seguridad y servicios".

\section{Medodología}

Existe cierto número de precedentes aplicados a la realidad argentina que, a partir de información censal y aplicando técnicas de análisis factorial, buscan identificar las dimensiones que subyacen en la distribución espacial de la población. La mayor parte de ellas se circunscriben a un único momento censal -de forma mayoritaria el de 2001 (excepciones son Mignone, 2009; Perren, 2014; Prieto y Formiga, 2012, o Valdés y Koch, 2009)-, y a un único aglomerado, aunque disponemos de algunas que atienden a dos o más ciudades, empleando las mismas variables en todas ellas (Gómez y Natera, 2012 y Natera, 2010, ambos con datos del Censo de 2001), o bien utilizando indicadores comparables, al pertenecer las ciudades objeto de estudio a países diferentes (Valdés y Koch, 2009).

La unidad de referencia espacial es en la práctica totalidad de los casos el radio censal y, pese a que el número de variables empleadas varía (entre un mínimo de 8 -Kanitscheider, 2007-y un máximo de 24 -Prieto 2012, y Prieto y Formiga, 2012-), estas suelen estar encuadradas en 4 o 5 grupos temáticos, siendo la estructura de la población y las características de las viviendas los más comunes. También hay una gran coincidencia en el instrumento estadístico empleado, que es el análisis factorial de componentes principales -análisis factorial exploratorio-, rotándose en la mayor parte de los casos la matriz de varianzas con el método varimax. Un último elemento común es que aportan la distribución espacial de los valores de los componentes detectados por el análisis factorial.

En nuestro caso, el volumen de variables disponibles para un estudio como el que nos ocupa es más reducido que el correspondiente a una investigación centrada en un único Censo, puesto que si pretendemos que los resultados sean comparables a lo largo del tiempo, las variables de partida deben ser las mismas en todos ellos. En este contexto, son varios los factores que inciden en la nómina de variables comunes potencialmente utilizables al nivel de desagregación espacial de radio censal. Por un lado, la amplitud temática de los cuestionarios censales, que ha variado de un Censo a otro, una limitación que afecta fundamentalmente a las características económicas de la población: 
es el Censo de 2001 el que más información aporta al respecto al nivel de radio censal, y el de 2010, con diferencia, el más parco, informando únicamente acerca de la condición de actividad de la población; ello hace imposible el empleo de variables de este tipo en nuestro estudio. Por otro, la forma de realización de la operación Censal marca la disponibilidad efectiva de variables; el empleo de métodos muestrales implicó el empleo en 1991 y 2010 de un cuestionario básico -aplicado a la totalidad de viviendas- y otro ampliado -implementado en un número sustancialmente menor de las mismas-, lo que limita dramáticamente las variables finalmente disponibles a nivel de radio censal.

De cualquier manera, hay todo un conjunto de variables definidas de igual manera en las tres operaciones censales, referidas tanto a la población como a los hogares y las viviendas, sobre las que es posible basar un estudio comparativo como el que nos ocupa, y de las que hemos seleccionado un total de 13, divididas en cinco grupos.

El primero de ellos está en relación con las características demográficas de la población: estructura por edad (porcentaje de población menor de 15 años, porcentaje de mayores), y relación de fecundidad, entendida como la proporción existente entre el número de menores de 5 años y las mujeres de entre 15 y 44 años.

Un segundo grupo de variables se centra en alguna de las características de la relación existente entre viviendas, hogares y población: cantidad de hogares por vivienda, como forma indirecta de medir los problemas de acceso a la vivienda por parte de la población; número de habitantes por vivienda, variable complementaria de la anterior y que permite, de forma indirecta, medir la presión que la población -y no los hogares- ejerce sobre el parque habitacional disponible; proporción de hogares que son ocupantes de la vivienda en la que residen por préstamo, por trabajo, o que se encuentran en "otra situación”, como medida indirecta de la incapacidad de obtener vivienda propia.

Las características de la instrucción de la población componen un tercer grupo de variables: grado máximo de instrucción "secundario incompleto" y "universitario completo", calculadas ambas sobre la población que no asiste pero asistió a un establecimiento escolar.

Un cuarto grupo corresponde a variables relacionadas con el origen de la población. Porcentaje de población nacida en país limítrofe y porcentaje de población nacida en país no limítrofe.

Por último, el quinto grupo contiene variables referidas a la pobreza estructural, concretamente: porcerntaje de casas A y departamentos sobre el total de viviendas; porcentaje de población con NBI retrete; y porcentaje de población que reside en viviendas inconvenientes, variable que, junto con la anterior, conforman los dos indicadores de Necesidades Básicas Insatisfechas de carácter universal.

Por último, indicar que el número de radios censales al que toda esta información queda referida es de 537 en 1991, 707 en 2001 y 744 en 2010.

\section{Resultados y discusión}

Para alcanzar nuestros objetivos, para responder la dos preguntas que nos hacíamos al comienzo, hemos aplicado a las 13 variables anteriores un análisis factorial de componentes principales, uno 
para cada operación censal. Previamente las variables fueron estandarizadas (obteniéndose con esta operación un conjunto de valores de media 0 y desviación estándar 1), y las matrices factoriales fueron rotadas según el método varimax. La solución factorial del análisis se ofrece en la tabla 1.

Tabla 1. Solución factorial del aglomerado del Gran San Miguel de Tucumán para los Censos de 1991, 2001 y 2010 (13 variables).

\begin{tabular}{|c|c|c|c|c|c|c|}
\hline Censo & 1991 & 1991 & 2001 & 2001 & 2010 & 2010 \\
\hline & $\begin{array}{c}\text { \% varianza } \\
\text { explicada }\end{array}$ & $\%$ acumulado & $\begin{array}{c}\text { \% varianza } \\
\text { explicada }\end{array}$ & $\%$ acumulado & $\begin{array}{c}\text { \% varianza } \\
\text { explicada }\end{array}$ & $\%$ acumulado \\
\hline $\begin{array}{c}\text { Componente } \\
1\end{array}$ & $45,7 \%$ & $45,67 \%$ & $31,7 \%$ & $31,7 \%$ & $45,3 \%$ & $45,3 \%$ \\
\hline $\begin{array}{c}\text { Componente } \\
2\end{array}$ & $19,2 \%$ & $64,9 \%$ & $30 \%$ & $61,7 \%$ & $15,1 \%$ & $60,4 \%$ \\
\hline $\begin{array}{c}\text { Componente } \\
3\end{array}$ & $9,1 \%$ & $74 \%$ & $9 \%$ & $70,7 \%$ & $12,9 \%$ & $73,3 \%$ \\
\hline $\begin{array}{c}\text { Componente } \\
4\end{array}$ & - & - & - & - & $9,8 \%$ & $83,1 \%$ \\
\hline
\end{tabular}

Fte: Censos de Población y Viviendas. Elaboración propia.

Como se puede observar en ella, el análisis ha diferenciado tres componentes en los dos primeros Censos y cuatro en el último. Entre todos explican una parte importante de la varianza original de las variables (columnas de porcentaje acumulado): más del 70\% en los Censos de 1991 y 2001, y más del $80 \%$ en el correspondiente a 2010. En la tabla 1 también se aprecia cómo no todos los componentes tienen la misma capacidad explicativa (columnas de porcentaje de varianza explicada), puesto que en los resultados de los tres Censos hay uno que explica una porción de la varianza inferior al $10 \%$, lo que le sitúa a gran distancia del primero de los componentes, que es el que, con diferencia, mayor capacidad explicativa tiene.

Seguidamente en la tabla 2 mostramos las estructuras factoriales; esto es, qué variables originales aportan más significado a cada uno de los componentes anteriores, y si su relación con ellos es positiva o negativa. Una estructura a partir de la cual es posible identificar de manera concreta las dimensiones que subyacen en la diferenciación de la población del Gran San Miguel.

Tabla 2. Estructura factorial del aglomerado del Gran San Miguel de Tucumán (1991-2010).

\begin{tabular}{|c|c|c|}
\hline \multicolumn{1}{|c|}{1991} & 2001 & 2010 \\
\hline \multicolumn{3}{|c|}{ Componente 1 } \\
\hline Menores de 15 años + & Menores de 15 años + & Menores de 15 años + \\
\hline Mayores de 64 años - & Mayores de 64 años - & Mayores de 64 años - \\
\hline Tasa de Fecundidad + & Tasa de Fecundidad + & Tasa de Fecundidad + \\
\hline \% Casas A y depart. - & \% Casas A y depart.- & \% Casas A y depart.- \\
\hline \% pobl. con NBI retrete + & \% pobl. con NBI retrete + & \% pobl. con NBI retrete + \\
\hline \% NBI vivienda inconv. + & \% NBI vivienda inconv.+ & \% NBI vivienda inconv.+ \\
\hline
\end{tabular}




\begin{tabular}{|c|c|c|}
\hline \% pobl. con sec. incomp + & \% pobl. con sec. incomp + \\
\hline \% nacidos en otro país - & \% pobl. con est. Super. - \\
\hline \multicolumn{3}{|c|}{ Componente 2} \\
\hline Habitantes/vivienda + & Habitantes/vivienda + & \% nacidos en pais limitr. + \\
\hline Hogares/vivienda + & \% nacidos en otro país - & \% nacidos en otro país - \\
\hline \% pobl. con est. Super. - & \% pobl. con sec. incomp + \\
\hline & \% pobl. con est. Super. - \\
\hline & Componente 3 \\
\hline \% nacidos en pais limitr. + & \% nacidos en pais limitr. + & Hogares/vivienda + \\
\hline Tenencia inconven. + & Tenencia inconv. + & Habitantes/vivienda + \\
\hline & Hogares/vivienda + & Componente 4 \\
\hline & \multicolumn{3}{|c}{} \\
\hline
\end{tabular}

+: saturaciones positivas en el componente. -: saturaciones negativas en el componente.

Fuente: Censos de Población. Elaboración propia.

La observación de dicha tabla muestra que la nómina de variables que aportan significado al Componente 1 se ha mantenido muy estable a lo largo de estos 20 años: las indicativas de la estructura demográfica de la población (juventud con signo positivo, envejecimiento negativo) y de las condiciones materiales de habitabilidad (positivas las indicativas de situaciones problemáticas como presencia de NBI-, negativas el resto) conforman en los tres censos el núcleo de su significado; se completan con las variables de instrucción en el Censo de 2010 (mejores niveles con signo negativo, niveles de instrucción más bajos positivo), y con una relativa al origen de la población, en el de 1991. Así pues, de manera global, este primer componente podría identificarse con "Estructura poblacional joven con problemas habitacionales", con un matiz de bajos niveles de instrucción en 2010.

Un segundo componente común en los tres censos ha sido también identificado: el número 2 en 1991 y 2001, y el número 3 en 2010. Son las variables indicativas de hacinamiento las que marcan su significado de manera evidente en 1991 y 2010, a las que se añaden las relativas al nivel de instrucción de la población en 1991 y 2001 y, en este último, también el origen foráneo de la misma. Por tanto, podría identificarse de manera global con "Presión sobre el parque habitacional”, con un matiz de bajos niveles de estudio de la población en 1991 y 2001.

La combinación de variables que aportan significado al resto de componentes (tercero en 1991 y 2001, segundo y cuarto en 2010) están indicando una situación de problemas de tenencia combinada con la presencia de inmigrantes provenientes de países limítrofes en 1991 y 2001. Una situación que el análisis ha fragmentado en 2010 en dos componentes, el segundo (identificado con inmigración) y el cuarto (identificado con problemas de tenencia de la vivienda).

Recapitulando: la estructura de las dos dimensiones principales que subyacen en la varianza de las 13 variables empleadas en nuestro estudio es extremadamente similar en los tres censos; presencia de población joven con problemas habitacionales, en primer lugar, presencia de presión poblacional sobre el parque de viviendas, en segundo. Y, por su parte, el resto de variables han sido agrupadas 
en un solo componente en 1991 y 2001, y en dos en 2010. Por tanto, podemos concluir que las dimensiones que gobiernan la diferenciación residencial del Gran San Miguel de Tucumán desde 1991, e identificadas a partir de 13 variables censales, se han mantenido bastante estables tanto en número como en significado en el periodo de estudio.

Una vez identificadas las dimensiones que gobiernan la distribución socio habitacional de la población en el Gran San Miguel de Tucumán, según resulta de la información censal empleada, las puntuaciones que alcanzan en los radios censales pueden ser cartografiados, ganando en riqueza el análisis. Por razones de espacio, nos centraremos en las correspondientes a los dos componentes que más varianza explican. En las figuras 2 a 4 se muestra la distribución de las puntuaciones que alcanzan los radios en el primer componente, "Estructura poblacional joven con problemas habitacionales”.

Figura 2. Puntuaciones de los radios en el componente Estructura poblacional joven con problemas habitacionales, Censo de 1991

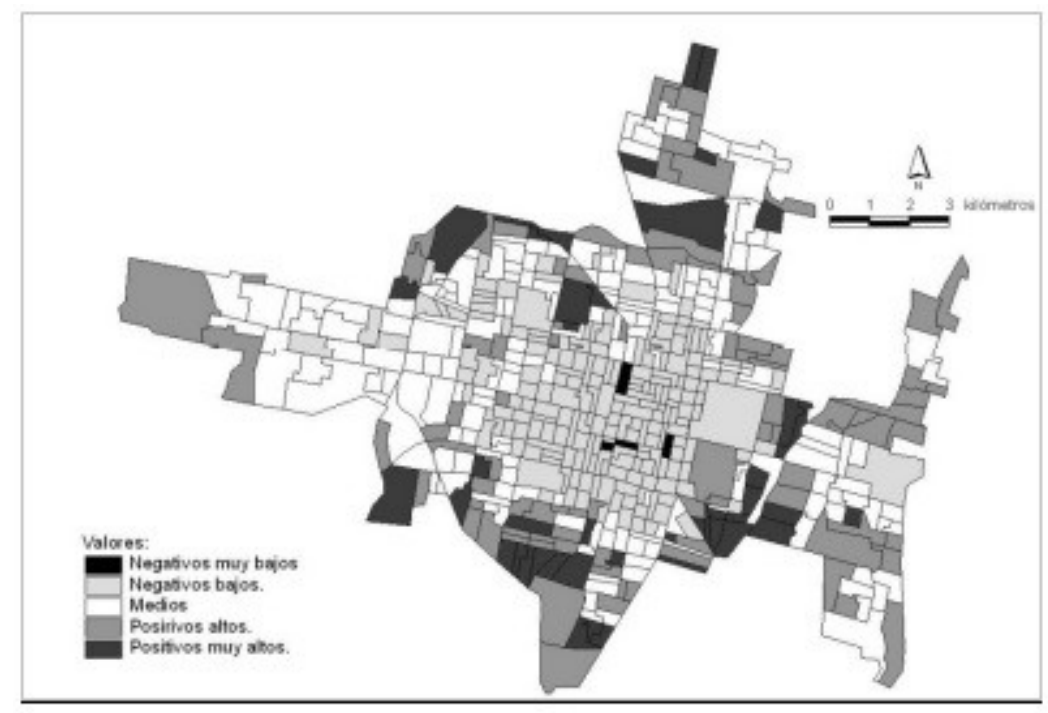

Elaboración propia. 
Figura 3. Puntuaciones de los radios en el componente Estructura poblacional joven con problemas habitacionales, Censo de 2001.

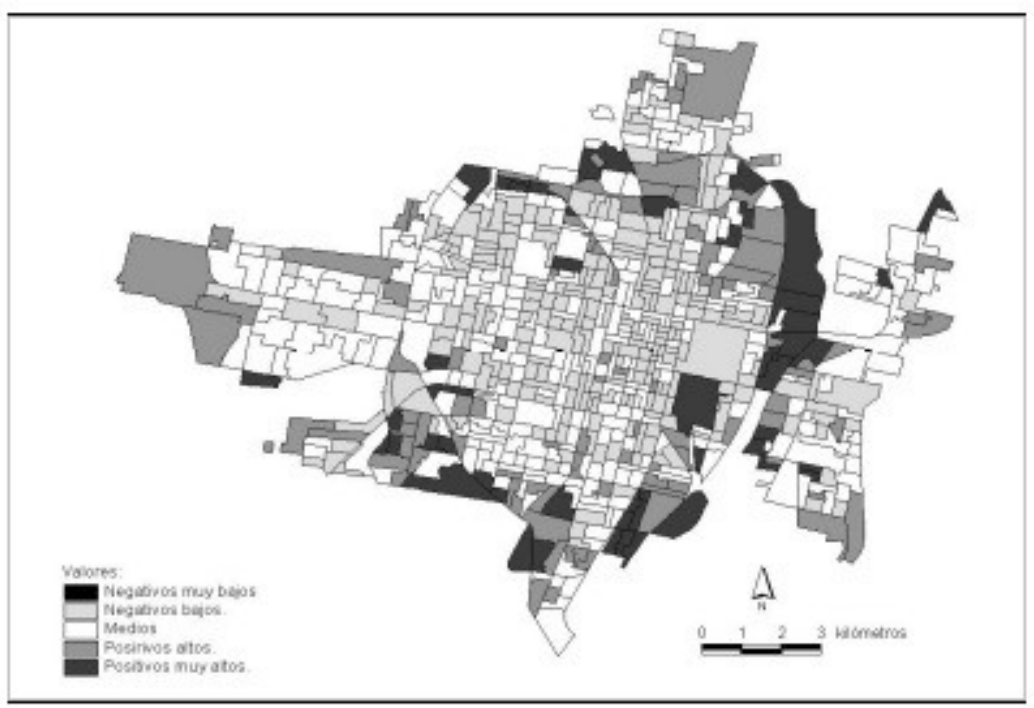

Elaboración propia.

Figura 4. Puntuaciones de los radios en el componente Estructura poblacional joven con problemas habitacionales, Censo de 2010.

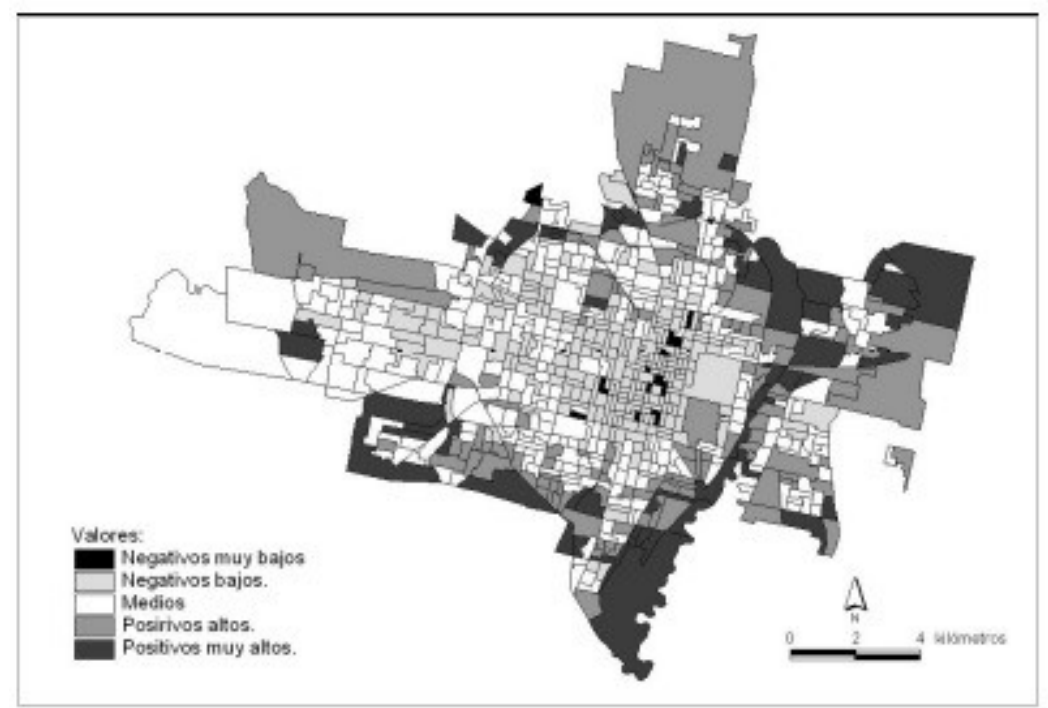

Elaboración propia.

En ellas se puede observar claramente cómo en los tres censos los radios censales con puntuaciones positivas muy altas o altas se localizan, de manera mayoritaria, en la periferia de la localidad de San Miguel de Tucumán y de las localidades menores (Alderetes, Banda del Río Salí, Villa Mariano Moreno y Yerba Buena). En San Miguel este conjunto de radios muestra un importante grado de contigüidad espacial, conformando una orla tan sólo rota en el límite con Yerba Buena, al oeste. En este sentido, la comparación de la cartografía de los tres censos muestra claramente cómo la expansión espacial en los extremos este y sur ha estado dominada por la presencia de radios con elevadas puntuaciones positivas en este componente, consecuencia de que, efectivamente, el 
crecimiento urbano aquí ha tenido como protagonista a barrios con calidades residenciales deficientes (Boldrini y Gómez, 2014). Es más, el Plan Estratégico de Tucumán define a San Miguel como "una ciudad de pobreza extendida y carácter estructural”, en la que "su situación social se presenta en el territorio de la ciudad de una manera diferenciada y contrastante según la zona que se trate” (Salas, 2016, p. 8).

La ciudad se presenta con un sector más consolidado (el área central contenida dentro de las cuatro avenidas principales) con mayores niveles de ocupación y de dotación de servicios, mientras que por fuera de este sector las expansiones [...] presentan evidencias de un profundo deterioro en la calidad del espacio urbano, situaciones de irregularidad dominial y carencia de infraestructuras (Salas, 2016, p. 10).

También se aprecia en esas mismas figuras cómo los radios con puntuaciones positivas altas o muy altas presentan "intrusiones” hacia el interior del aglomerado; la más importante y estable en los tres censos es la localizada en el entorno de Villa Muñecas. Esta zona presenta un déficit de vivienda derivado del rápido aumento de la población y de una escasa oferta de vivienda para una población de bajos recursos con dificultades para acceder vía financiamiento a una vivienda propia (Centeno, 2013). En ella conviven áreas de vivienda formal con otras procedentes de invasiones, como los barrios Juan XXIII o Juan Pablo II. Surgidos de un proceso de urbanización irregular, su progresiva densificación dio lugar a la ocupación de tierras colindantes aún libres, apareciendo nuevos barrios (Del Castillo, 2014, p. 6), alguno de ellos bautizados como "ampliaciones" Ampliación Juan XXIII-. Se trata, por tanto, de una realidad no resuelta en los últimos 20 años, y que ha sido registrada en las tres operaciones censales y detectada por el análisis.

En contraste, los radios que se extienden por el centro del aglomerado tienen en los tres censos valores negativos bajos o muy bajos, tal y como cabría esperar de la zona con mayor dotación infraestructural y de servicios, y en la que reside la población más envejecida. Al mismo tiempo, conforme avanzamos en el tiempo, puede comprobarse cómo se va consolidando una extensión lineal de radios con puntuaciones bajas dirigida hacia la localidad de Yerba Buena, asimilable a las expansiones lineales de población de clase alta postulada por los modelos a los que anteriormente hicimos referencia, y que también han sido identificadas en otras ciudades argentinas. En este sentido, se ha indicado (Malizia y Boldrini, 2012) que a partir del 2000 se registró un crecimiento exponencial de urbanizaciones cerradas en Yerba Buena, siendo esta la zona elegida mayoritariamente por los grupos de elevado poder adquisitivo para instalar sus residencias. En contraste, la presencia de asentamientos informales en ella es muy escasa, así como también lo son las actuaciones de obra pública para sectores pobres (Gómez, Cuozzo y Boldrini, 2015). Todos estos elementos explican la presencia, y consolidación, de esta área secundaria de población más envejecida y sin problemas habitacionales que ha detectado el análisis.

Por su parte, la distribución espacial del componente “presión sobre el parque habitacional”, es mostrada en las figuras 5 a 7. En ellas se aprecia cómo, una vez más, de manera sistemática, los radios correspondientes al centro del aglomerado y a la expansión hacia Yerba Buena obtienen valores muy bajos o bajos, a los que se unen algunos localizados en la periferia oriental. Puede parecer contradictorio que dos áreas tan diferentes desde el punto de vista de la población y las características de las viviendas compartan puntuaciones en este componente. Esto se explica por el 
hecho de que la periferia oriental del aglomerado, colindante con el contaminado curso del Río Salí, es asiento de asentamientos informales de reciente aparición (Gómez y Boldrini, 2014), por lo que no están afectados por el proceso de densificación al que en los párrafos anteriores hicimos referencia. Esta ausencia de densificación es el elemento que comparten con el centro y Yerba Buena, y es lo que está poniendo de manifiesto el componente.

Figura 5. Puntuaciones de los radios en el componente Presión sobre el parque habitacional, Censo de 1991.

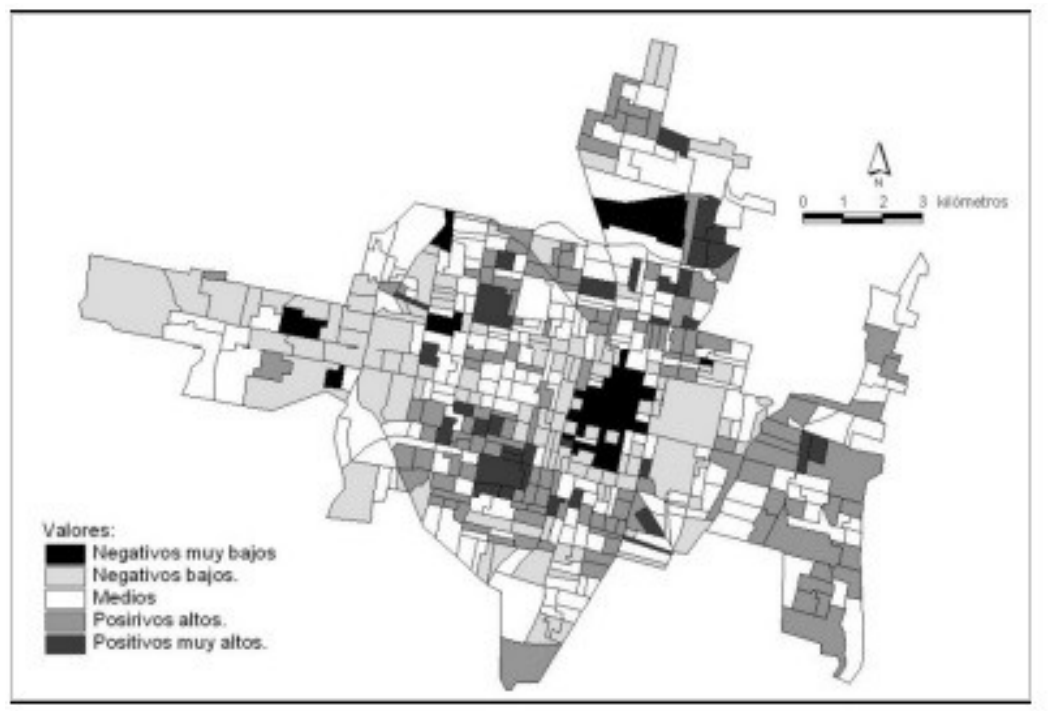

Elaboración propia.

Figura 6. Puntuaciones de los radios en el componente Presión sobre el parque habitacional, Censo de 2001.

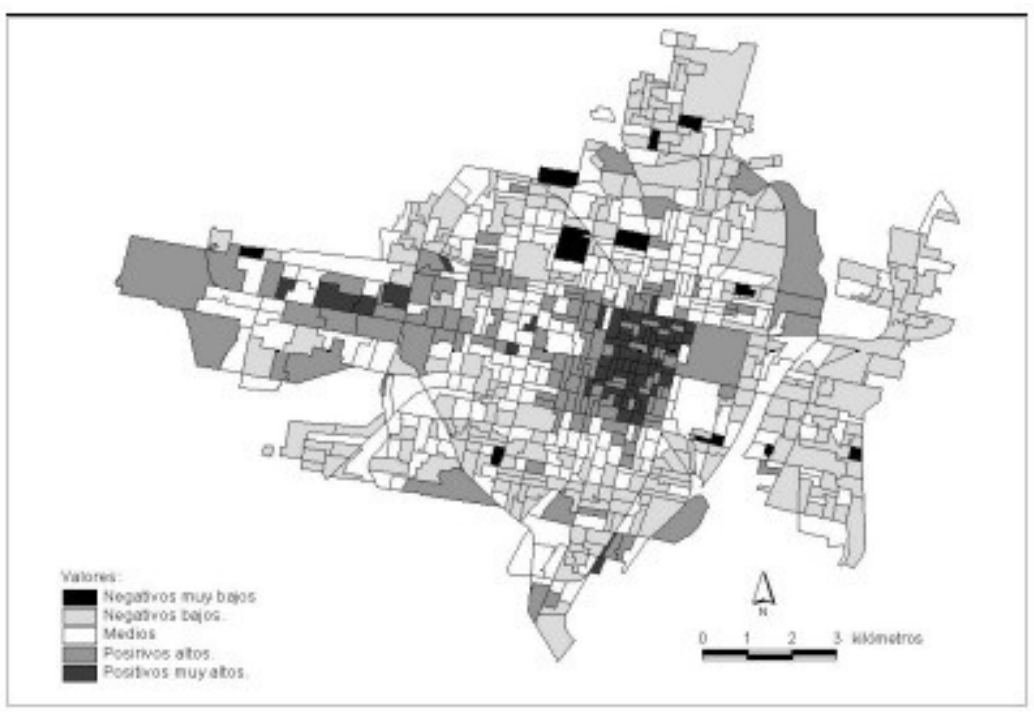

Elaboración propia. 
En el otro extremo de la escala, es interesante indicar que los radios que obtienen las puntuaciones positivas más elevadas no están en la periferia extrema, sino en zonas pericentrales del aglomerado, correspondiéndose con alguna de las concentraciones más importantes de villas de emergencia que ya estaban presentes desde al menos la década de los ochenta, y en cuyo entorno inmediato estaban surgiendo nuevos asentamientos informales a mediados de la década de los noventa; es el caso del área de 11 de Marzo, al sur de Juan XXIII al norte. En párrafos anteriores habíamos indicado que estas áreas se fueron densificando con el tiempo -esto es, aumentando el número de habitantes y hogares por vivienda-, de ahí los importantes valores positivos que obtenían en 1991. Las salidas de estos “excedentes” poblacionales hacia otras áreas del aglomerado disminuyó en ellas la presión sobre el parque habitacional, haciendo descender, por tanto, los valores del componente en el siguiente Censo. Además, la exclusión de las variables relacionadas con el nivel de instrucción de la población en el censo de 2011 de la nómina que da significado a este componente, hizo disminuir aún más sus valores.

Figura 7. Puntuaciones de los radios en el componente Presión sobre el parque habitacional, Censo de 2010.

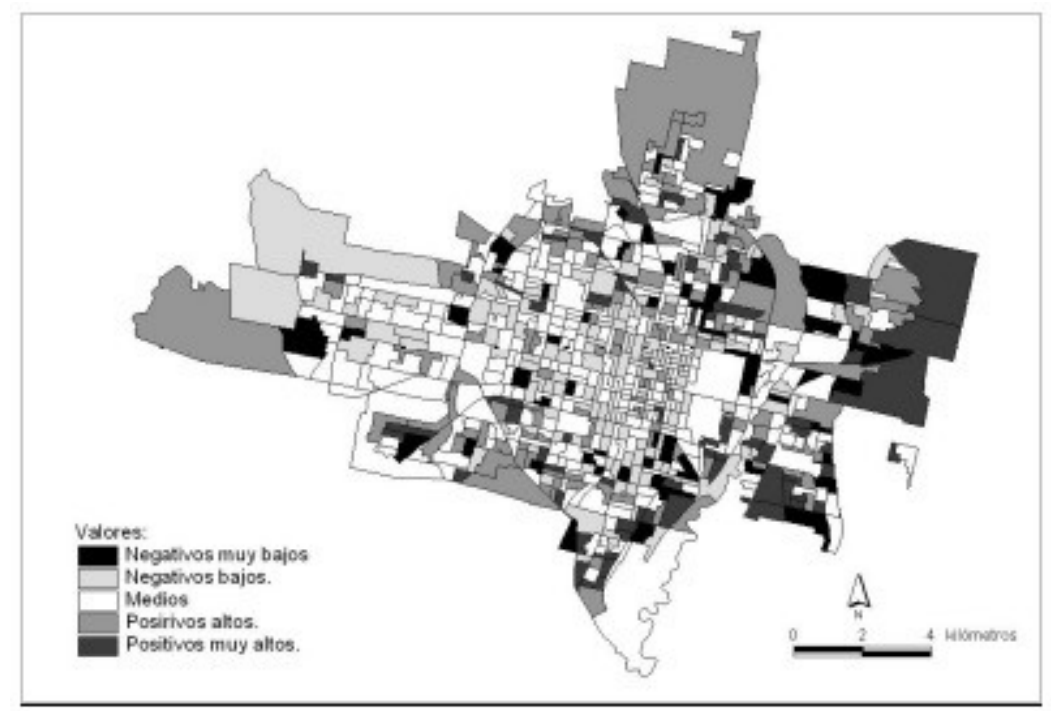

Elaboración propia.

\section{Conclusiones}

El proceso de crecimiento del Gran San Miguel de Tucumán desde los noventa, en un contexto de progresiva retirada del Estado como ente productor de vivienda, ha venido desarrollándose entre dos polos opuestos: por un lado, tejido urbano correspondiente a viviendas con comparativamente elevadas calidades constructivas e infraestructurales, contenidas en urbanizaciones cerradas; por otro, asentamientos de vivienda popular, no siempre dominialmente regularizados y sistemáticamente peor dotados que los anteriores. Una dualidad que tiene reflejo espacial, siendo la localidad de Yerba Buena la que concentra la mayor parte de las urbanizaciones cerradas, las áreas periféricas del aglomerado la mayor parte de los asentamientos. Esta realidad, fácilmente identificable para el observador casual, puede considerarse como la manifestación visible de causas 
más profundas que afectan a cómo se distribuye espacialmente la población del aglomerado, una población heterogénea en lo tocante a su estructura por edad, niveles de instrucción o características económicas, entre otras. $\mathrm{Y}$ es a través del empleo de herramientas estadísticas, aplicadas a información censal muy desagregada espacialmente, como se pueden identificar esas causas profundas y seguir su evolución temporal. No obstante, debe tenerse en cuenta la dificultad que impone la selección de variables, puesto que estas deben ser comparables a lo largo de las tres rondas censales que van de 1991 a 2010.

En el caso del Gran San Miguel, según se desprende de las 13 variables que hemos empleado, las causas subyacentes en la distribución espacial de la población durante el reciente proceso de crecimiento del aglomerado se han mantenido estables: son, básicamente, por un lado, una dualidad entre un grupo de población con estructura de edad joven y con problemas habitacionales y otro más envejecido y en mejores condiciones de vivienda; por otro, la presencia de presión sobre el parque habitacional. La distribución espacial de la primera de las dimensiones muestra cómo las sucesivas periferias que surgían del crecimiento del aglomerado obtenían puntuaciones positivas altas o muy altas en ellas; esto quiere decir que la expansión del Gran San Miguel ha estado protagonizada por población joven afectada por problemas habitacionales. Esta situación no es nueva $y$, como en su momento vimos, ha sido puesta de manifiesto por otras investigaciones; pero lo que resulta preocupante, desde el punto de vista de la planificación, es que se haya mantenido estable durante los últimos 20 años. En contraste, nuestro análisis muestra la progresiva consolidación de un eje que, partiendo desde el centro del aglomerado, toma dirección oeste hacia Yerba Buena y tiene como protagonista al grupo opuesto al anterior. De ello puede deducirse que el proceso de crecimiento del Gran San Miguel está provocando una progresiva fragmentación, muy marcada espacialmente y que, lejos de revertirse, se está consolidando, según se deduce de la información censal empleada.

En cuanto a la distribución espacial de la segunda dimensión, la presión sobre el parque habitacional, es sistemáticamente el área central del aglomerado el que menor afectación presenta, acorde con una estructura por edad más envejecida y los precios de la vivienda más elevados. No es, sin embargo, la periferia extrema la que presenta los valores positivos más elevados en esta dimensión, sino las áreas intermedias del aglomerado, localizadas entre el centro y la periferia existente en cada uno de los tres años analizados. Esto es reflejo de la demanda de vivienda existente; una demanda de vivienda que es satisfecha en el mercado legal tan sólo por parte de los hogares que cuentan con los recursos económicos necesarios, y en el informal por el resto, siendo la responsable no sólo del crecimiento espacial del Gran San Miguel, sino de las características que este toma y que ya fueron indicadas en los epígrafes anteriores.

El mapa social que se deriva de la aplicación del análisis factorial no ha variado en su esencia, por tanto, en nuestro periodo de estudio. Sí acaso los procesos de fragmentación urbana que ya fueron detectados por otros investigadores (GEO, 2006; Gómez, Cuozzo y Peralta, 2015) se han agudizado. Un efecto del contexto neoliberal en el que se ha insertado el crecimiento urbano de las metrópolis latinoamericanas (Pradilla, 2014) que ha quedado reflejado en las distribuciones de las puntuaciones de las dos principales dimensiones que subyacen en la diferenciación residencial del Gran San Miguel. 


\section{Bibliografía}

Bähr, J. \& Mertins, G. (1982). A model of the social and spatial differentiaion of Latin American Metropolitan Cities. Appplied Geography and Development, 19, 22-45.

Boldrini, P, y G ómez López, C. (2014). Participación popular en la producción del hábitat. El caso del Área Metropolitana de Tucum án-Argentina. Revista de Geografía Norte Grande, 57, 67-82. doi: http://dx.doi.org/10.4067/S0718-34022014000100006

Borsdorf, A. (2003). Cómo modelar el desarrollo y la dinámica de la ciudad latinoamericana. EURE, 29(86), 37-49. Recuperado de http://www.eure.cl/index.php/eure/article/view/1265/362

Buzai, G. (2014). Mapas sociales urbanos. Buenos Aires: Lugar Editorial.

Centeno, R. N. (2013). Informalidad en el proceso de urbanización de Villa Muñecas (Tucumán, Argentina). GeoGraphos, 4(34), 52-68. doi: http://dx.doi.org/10.14198/GEOGRA2013.4.34.

Del Castillo, A. (2014). Un estudio de caso sobre la pobreza urbana en Gran San Miguel de Tucumán (Argentina) en los inicios del siglo XXI. III Jornadas Nacionales sobre estudios regionales y mercados de trabajo. Universidad Nacional de Jujuy. Recuperado de http://www.aacademica.org/iii.jornadas.nacionales.sobre.estudios.regionales.y.mercados.de.trabajo/ $\underline{21}$

GEO (2006). San Miguel de Tucumán. Perspectivas del Medio Ambiente Urbano. Recuperado de http://www.pnuma.org/deat1/pdf/2007GEOSanMigueldeTucumn.pdf

Griffin, E. \& Ford, L. (1980). A model of Latin American city structure. Geographical Review, 70(4), 397-422.

Gómez, N. Javier y Natera Rivas, J. J. (2012). Diferenciación residencial de los aglomerados mayores de la región centro de Argentina. Cuadernos de Geografía. Revista Colombiana de Geografía, 21(1), 11-28. Recuperado de http://www.revistas.unal.edu.co/index.php/rcg/article/view/23530/36754

Gómez López, C., Cuozzo, R. L. y Boldrini Peralta, P. (2015). Impactos de las políticas públicas de hábitat en la configuración del espacio urbano entre 2003 y 2013- El caso del área metropolitana de Tucumán, Argentina. Cuaderno Urbano. Espacio, Cultura y Sociedad, 19, 153-178. Recuperado de http://arq.unne.edu.ar/publicaciones/cuaderno urbano/cu 19/index.html

Howell, D. C. (1989). A model or argentine city structure. Revista Geográfica, 109, 129-140.

Janoschka, M. (2002). El nuevo modelo de la ciudad latinoamericana: fragmentación y privatización. EURE, 28(85), 11-20. doi: http://dx.doi.org/10.4067/S0250-71612002008500002.

Kanitscheider, S. (2007). Diferenciación socioespacial en la periferia argentina, el ejemplo de San Salvador de Jujuy. Revista de Geografía Norte Grande, 37, 23-33. doi: http://dx.doi.org/10.4067/S0718-34022007000100002

Malizia, M. y Boldrini Peralta, P. (2012). Las lógicas de ocupación del espacio urbano. Un estudio de realidades contrapuestas. El caso de las urbanizaciones cerradas y villas miseria en Yerba Buena, Gran San Miguel de Tucumán. Cuadernos FHyCS-UNJu, 41, 197-219. Recuperado de 
http://revista.fhycs.unju.edu.ar/index.php/cuadernos/article/view/177/118

Mertins, G. (1995). La diferenciación socioespacial y funcional de las ciudades intermedias latinoamericanas: ejemplos del noroeste argentino. Revista Interamericana de Planificación, 28(112), 55-68.

Mignone, A. M. (2011). La pobreza urbana en las capitales provinciales del Norte Grande Argentino. Cuaderno Urbano. Espacio, cultura, sociedad, 10, 63-81. Recuperado de http://arq.unne.edu.ar/publicaciones/cuaderno urbano/cu 10/index.html

Ministerio del Interior, Obras Públicas y Vivienda (2016). Atlas ID. Recuperado de http://atlasid.planificacion.gob.ar/default.aspx

Perren, J. (2014). Diferenciación socio-residencial en las ciudades intermedias argentinas. El caso de Neuquén hacia comienzos de los noventa. Revista Geográfica Digital IGUNNE, 11(21), 1-18. Recuperado de http://hum.unne.edu.ar/revistas/geoweb/Geo21/contenidos/perren14.htm

Prieto, M. B. (2012). Segregación socio-residencial en ciudades intermedias. El caso de Bahía Blanca, Argentina. Breves Contribuciones del Instituto de Estudios Geográficos, 23, 129-156. Recuperado de http://ojs.filo.unt.edu.ar/index.php/bcieg/article/view/157/136

Prieto, M. B. y Formiga, N. (2012). Segregación socio-espacial en ciudades intermedias. El caso de Bahía Blanca -Argentina-. XII Seminario Internacional. Red Iberoamericana de Investigadores en Globalización y Territorio. Belo Horizonte, Brasil, 1 a 5 de octubre de 2012. Recuperado de www.inegi.org.mx/eventos/2011/Conf Ibero/doc/ET4 19 PRIETO.pdf

Salas, I. (dir.) (2016). Plan estratégico urbano territorial para la ciudad de San Miguel de Tucumán. Recuperado de http://www.sanmigueldetucuman.gov.ar/bajar/PlanSMT2016.pdf.

Timms, D. (1976). El mosaico urbano. Hacia una teoría de la diferenciación residencial. Madrid: IEAL.

Valdés, E. G. y Koch, M. R. (2009). Tendencias de segregación residencial en metrópolis latinoamericanas intermedias al inicio del siglo XXI. Porto Alegre (Brasil) y Córdoba (Argentina). Revista Líder, 15(11), 85104. Recuperado de http://ceder.ulagos.cl/lider/images/numeros/15/[LIDERVol15A\%C3\%B1011-2009ISSN-0717-0165]4.-Tendenciasdesegregaci\%C3\%B3nresidencialenmetr\%C3\%B3polis.pdf 